\title{
An Optimization Method of Deterministic Measurement Matrix in Distributed Compressed Video Sensing
}

\author{
Jiawei Qin, Dengyin Zhang* and Liang Xie \\ Nanjing University of Posts and Telecommunications \\ Nanjing, China \\ ${ }^{*}$ Corresponding author
}

\begin{abstract}
This electronic Compressed Sensing (CS) is a new theoretical framework for information acquisition and processing, which provides a new way for signal sampling. In order to solve the problem of the constraints of traditional Nyquist sampling theorem, CS based on the sparsity of signal, randomness of the measurement matrix and nonlinear optimization algorithm can achieve the compression and reconstruction of the signal. In the process of compressive sensing, the measurement matrix plays an important role in signal sampling and reconstruction. This construction is based on the orthogonal symmetric Toeplitz matrix in this paper. The pseudorandom feature of the deterministic measurement matrix is improved by pseudorandom loop construction method to ensure the random performance of the measurement matrix and optimize the compression measurement effect.
\end{abstract}

Keywords-compressed sensing; orthogonal symmetric toeplitz matrix (OSTM); pseudorandom

\section{INTRODUCTION}

In comprehensive sensing, the measurement matrix plays a crucial role in acquisition and reconstruction of the signal. It can make any sparse signal keep its main information not lost when projecting from high-dimensional space to low-dimensional space and ensure accurate reconstruction of the original signal. It has been pointed out that a good measurement matrix should require fewer measurement samples at the same sparsity, which is easy to implement in hardware and optimization of algorithm and has universal characteristic in the literature. It has been proved that random measurement matrices (including Gaussian random matrices, Bernoulli random matrices, etc.) satisfy RIP characteristics [1] and can be used as a universal measurement matrix. However, due to the randomness of this matrix is too strong, such measurement matrix is not practical both in the hardware implementation and algorithm reconstruction.

In order to overcome the shortcomings of the above matrix, we choose orthogonal symmetric Toeplitz matrix as the measurement matrix and increase the pseudorandom characteristics of the deterministic measurement matrix by introducing a method of pseudorandom loop construction.

\section{DISTRIBUTED COMPRESSIVE VIDEO SENSING}

Let $\mathrm{x}$ be a one-dimensional signal of length $\mathrm{N}$, there is a set of orthogonal basis vectors $\Psi$ such that $\mathrm{x}=\Psi \theta$, where $\Psi \mathrm{N} \times \mathrm{N}$ is a sparse basis matrix, there are only $k$ large coefficients or $k$ non- zero coefficients in $\theta$, then $\mathrm{x}$ is called compressible or k-sparse. It has been pointed out that the signal can be measured for compression by using a matrix $\Phi_{\mathrm{M} \times \mathrm{N}}(\mathrm{M}<<\mathrm{N})$ that is not related to $\Psi$ in compressive sensing theory which is

$$
y=\Phi_{M \times N} x=\Phi \Psi \theta=A^{C S}
$$

Where $\mathrm{y}$ is the measured value, $\mathrm{ACS}=\Phi \Psi$ is a $\mathrm{M} \times \mathrm{N}$ random matrix. From the RIP theory, as long as the $\Phi \Psi$ satisfies the RIP characteristic, we can reconstruct the $\mathrm{K}$ maxima of the $\mathrm{N}$ dimensional signal by $\mathrm{K} \lg (\mathrm{N} / \mathrm{K})$ measurement value which is by solving the L1 norm constraint optimization problem

$$
\begin{aligned}
& \hat{\theta}=\arg \min \|\theta\|_{1} \\
& \sigma . \tau=\Phi \Psi \theta
\end{aligned}
$$

In order to get the sparse representation of $\mathrm{x}$, the original signal is reconstructed accurately by the transform basis $\Psi$ as follows

$$
\hat{x}=\Psi \hat{\theta}
$$

It has been established that random matrices whose entries are drawn independently from certain probability distributions satisfy RIP with overwhelming probability [2]-[4]. However, such random matrices are impractical for large $\mathrm{N}$, since they require high computational and storage complexity.

Deterministic measurement matrix is an important type of matrix in practical application of compressive sensing. At present, proof of the RIP nature of such matrix is still a challenging issue. For the analysis of the properties of deterministic measurement matrix, many literatures have proposed the statistical definition SRIP of RIP property. It is pointed out that under the condition of satisfying SRIP, the matrix can also be used as the measurement matrix of compressive sensing and achieve good reconstruction results.

\section{OPTIMIZATION DESIGN BASED ON OSTM}

Orthogonal symmetric Toeplitz measurement matrix $(\mathrm{OSTM})$ is a deterministic measurement matrix which is 
proposed by Bottcher [5] in compressed sensing, the matrix is constructed as follows:

\section{A. Use a Binary Sequence of N/2 Length as the Sequence of Numbers}

$$
\sigma=\left[s_{1}, \ldots, s_{N / 2}, \pm s_{1}, s_{N / 2}, \ldots, s_{2}\right]
$$

At the same time, performs inverse fast Fourier transform (IFFT) on the symbol sequence to obtain a g-sequence of length N.

$$
g=\operatorname{IFFT}(\sigma)
$$

B. Use the Elements of Gas the First Row of Orthogonal Symmetric Toeplitz Matrix, and Follow the Rules of Circulation to Construct $N \times N$ Phalanx $\Phi$.

C. Randomly Select $M$ Rows in $\Phi$ and Normalize Them, At Last We Obtain the Final Orthogonal Symmetric Toeplitz Measurement Matrix

$$
\Phi_{N}=\left(\begin{array}{cccccccccc}
a & b & c & \ldots & f & g & f & \ldots & c & b \\
b & a & b & c & \ldots & f & g & f & \ldots & c \\
c & b & a & b & c & \ldots & f & g & f & \ldots \\
\vdots & & & & & & & & & \vdots \\
f & & & & & & & & & \ldots \\
g & f & & & & & & & & \ldots \\
f & g & f & & & & & & & \ldots \\
\vdots & & & & & & & & & \vdots \\
b & c & & & & & & & & \ldots
\end{array}\right)
$$

After the second step, it can be proved that the $\mathrm{N} \times \mathrm{N}$ matrix $\Phi \mathrm{N}$ is orthogonal and Toeplitz. The sign of s1 in the middle of $\sigma$ depends on the parity of the location of $\varphi 1$, which is the largest value in $g$. The specific structure of the sign sequence is a requirement of OSTM. Since there are $2 N / 2$ binary sequences of length $\mathrm{N} / 2$, clearly some are better than others. The bound based on Chebyshev's inequality was too loose to capture the impact of $s$ [7]. In this paper, we will significantly improve the bound by exploiting Stein's method [5]. This will enable us to achieve near-optimal performance by using a Golay's complementary sequence as $\mathrm{s}$ of the sign sequence. The specific steps of the method are as follows:

Step 1: The Golay's complementary sequence is selected as a symbol sequence, and $N n \times n$ square matrices $\Phi_{i}(1 \leq i \leq N)$ are generated according to the construction rule of OSTM, and each $\Phi_{\mathrm{i}}$ is an orthonormal Symmetric Toeplitz matrix.

Step 2: The generated $\mathrm{N}$ square matrices are regarded as a one-dimensional sub-block row vector $\left(\Phi_{1}, \Phi_{2}, \ldots, \Phi_{\mathrm{N}}\right)$, and then $\mathrm{N}-1$ remaining row vectors are generated according to the construction method of the circular matrix, and finally a square matrix $\Phi$ of the sub-block matrix $\mathrm{Nn} \times \mathrm{Nn}$ is obtained, which is

$$
\Phi=\left(\begin{array}{ccccc}
\Phi_{N} & \Phi_{N-1} & \ldots & \Phi_{2} & \Phi_{1} \\
\Phi_{1} & \Phi_{N} & \ldots & \Phi_{3} & \Phi_{2} \\
\vdots & \vdots & \vdots & \vdots & \vdots \\
\Phi_{N-1} & \Phi_{N-2} & \ldots & \Phi_{1} & \Phi_{N}
\end{array}\right)
$$

Step 3: Select any $M$ line from the square $\Phi$ as the measurement matrix, and normalize it, the final measurement matrix

$$
\Phi_{M}=\sqrt{N \mathrm{n} / M} \Phi
$$

Specific optimization process shown in Figure I:

Using the Golay's complementary sequence $\sigma$ as a symbol sequence, $g$ is used as the first row element of the orthogonal Symmetric Toeplitz matrix according to the Inverse Fourier Transform, $g=\operatorname{IFFT}(\sigma)$, and then the orthogonally symmetric Toeplitz matrix (OSTM) is constructed according to the construction method $\mathrm{N}$ square matrix $\Phi_{i}$, where $1 \leq i \leq N$

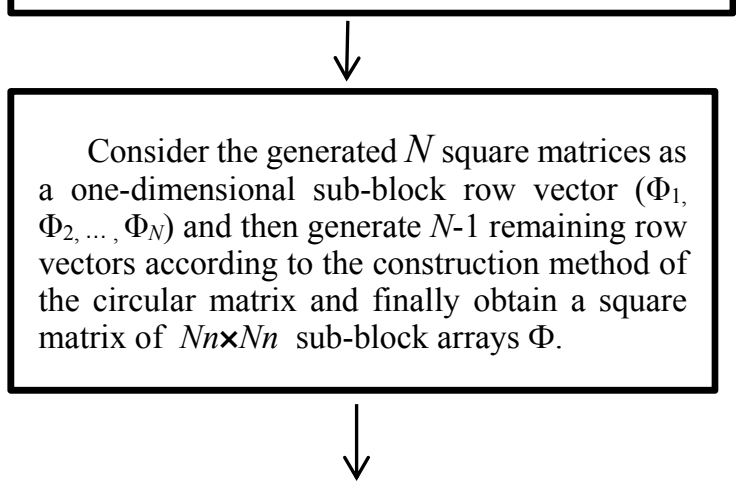

Finally, randomly select $M$ row vectors from the block square $\Phi$ and multiply them by the coefficient $\sqrt{N n / M}$ to obtain the final measurement matrix $\Phi_{M}=\sqrt{N \mathrm{n} / M} \Phi$

\section{FIGURE. I. OPTIMIZED FLOW CHART BASED ON ORTHOGONAL SYMMETRY TOEPLITZ MATRICES (OSTM)}

The orthogonal symmetric Toeplitz matrix is used to optimize by introducing pseudorandom cycle. the optimized measurement matrix can satisfy the statistical RIP (constrained equidistance) theory with great probability and the reason for using the OSTM lies in that OSTM is easier to generate and more efficient than existing measurement matrices, because only $n$ elements need to be stored to implement the matrix. In addition, the structure of Toeplitz is well-suited for many applications such as channel estimation and system identification. 


\section{SIMULATIONS}

In this newly created file, highlight all of the contents and import your prepared text file. You are now ready to style your paper; use the scroll down window on the left of the MS Word Formatting toolbar. In order to test the performance of the algorithm proposed in this paper, the program is simulated on the MATLAB platform and compared with Bernoulli matrix and OSTM. The first 60 frames of Foreman in CIF format $(352 \times$ 288) are used as the test sequence frames and the picture of Lena, Coins, Boat $(256 \times 256)$ are used. The measured values is reconstructed by using BCS-SPL [9]. The criteria for evaluating the algorithm are the peak signal-to-noise ratio (PSNR), which reflects the objective non-key frame reconstruction quality, and reconstruction time (rec-time).

TABLE I. THE RECONSTRUCTED TIME OF DIFFERENT MEASUREMENT MATRIX (S)

\begin{tabular}{|c|c|c|}
\hline Bernoulli matrix & OSTM & Optimized OSTM \\
\hline 0.061430 & 0.011651 & 0.012876 \\
\hline
\end{tabular}

TABLE II. PSNR VALUES OF RECONSTRUCTED IMAGES FROM DIFFERENT MEASUREMENT MATRIX (UNIT: DB)

\begin{tabular}{|c|c|c|c|c|c|}
\hline Image & $\begin{array}{c}\text { Measurement } \\
\text { matrix }\end{array}$ & $\begin{array}{c}\text { Sampling } \\
\text { rate } \\
\mathbf{0 . 3 0}\end{array}$ & $\begin{array}{c}\text { Sampling } \\
\text { rate } \\
\mathbf{0 . 4 0}\end{array}$ & $\begin{array}{c}\text { Sampling } \\
\text { rate } \\
\mathbf{0 . 4 5}\end{array}$ & $\begin{array}{c}\text { Sampling } \\
\text { rate } \\
\mathbf{0 . 5 0}\end{array}$ \\
\hline \multirow{5}{*}{ Lenna } & Bernoulli matrix & 25.5356 & 27.3532 & 28.3515 & 29.0973 \\
& OSTM & 25.8376 & 27.6686 & 29.4388 & 29.3401 \\
& Optimized OSTM & 26.9482 & 29.1712 & 30.1771 & 31.2151 \\
\hline \multirow{5}{*}{ Coins } & Bernoulli matrix & 25.6017 & 27.8137 & 28.8099 & 29.6653 \\
& OSTM & 25.4333 & 27.5434 & 28.4813 & 29.2583 \\
& Optimized OSTM & 26.6295 & 28.8598 & 29.8257 & 30.3486 \\
& Bernoulli matrix & 21.8164 & 23.4061 & 24.1733 & 24.9178 \\
& OSTM & 22.4073 & 23.6190 & 24.5885 & 25.2457 \\
& Optimized OSTM & 23.8451 & 25.6417 & 26.3360 & 26.9386 \\
\hline \multirow{4}{*}{ Foreman } & Bernoulli matrix & 23.2159 & 25.1067 & 25.9067 & 26.6739 \\
& OSTM & 23.5081 & 25.2132 & 25.9286 & 26.8560 \\
& Optimized OSTM & 24.8003 & 27.1328 & 27.8387 & 28.1345 \\
\hline
\end{tabular}

Table I shows the construction time of different measurement matrix. From Table I, we can see that the rec-time of the Optimized OSTM is less than that of Bernoulli random matrix but is longer than that of OSTM. Table II lists PSNR of frames at different subrates. Figure III more naturally shows the average PSNR (avg-PSNR) of frames and images with the subrate changes. It can be seen from Table II and Figure III that PSNR of this paper's algorithm is the largest compared with other methods at the same sampling. The Optimized OSTM proposed in this paper can improve reconstructed frame quality. Avg-PSNR of this method is $1.8 \mathrm{~dB}$ higher than that of other schemes. When subrate is 0.5 , the PSNR of other schemes have a maximum of $29.3401 \mathrm{~dB}$, while the proposed method has a $1.875 \mathrm{~dB}$ improvement of $29.3401 \mathrm{~dB}$.

Figure II shows the subjective visual comparison chart of lena's reconstructed images by the methods. When the subrate is 0.4 , the PSNR of other schemes have a maximum of $27.6686 \mathrm{~dB}$, while this method is improved by $2.5085 \mathrm{~dB}$ to become 30.1771 $\mathrm{dB}$. This method clearly has the best subjective visual quality. This method clearly has the best subjective visual quality.

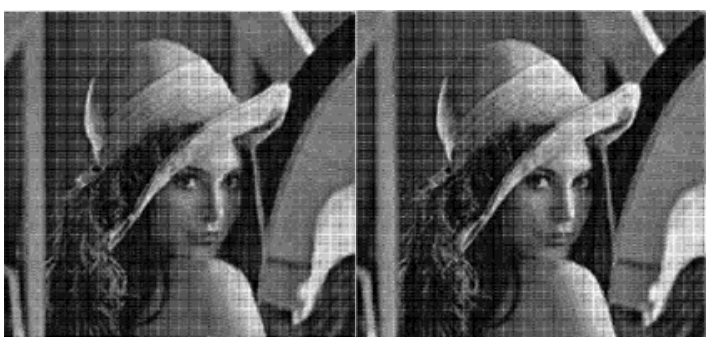

(A) (B)

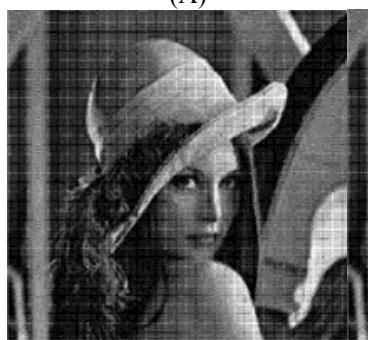

(C)

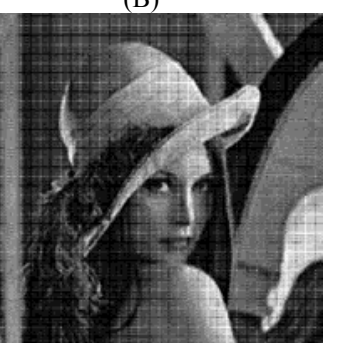

(D)
FIGURE II. THE SUBJECTIVE VISUAL COMPARISON CHART OF LENA'S RECONSTRUCTION BY DIFFERENT MATRIX WHEN THE SUBRATE IS 0.4: (A) ORIGINAL FRAME; (B) BERNOULI MATRIX; (C) OSTM; (D) OPTIMIZED OSTM. 
Figure III shows the subjective visual comparison chart of Foreman's second frame reconstructed by the methods. When the subrate is 0.4 , the PSNR of other schemes have a maximum of $25.2132 \mathrm{~dB}$, while this method is improved by $1.9196 \mathrm{~dB}$ to become $27.1328 \mathrm{~dB}$. This method clearly has the best subjective visual quality. This method clearly has the best subjective visual quality.

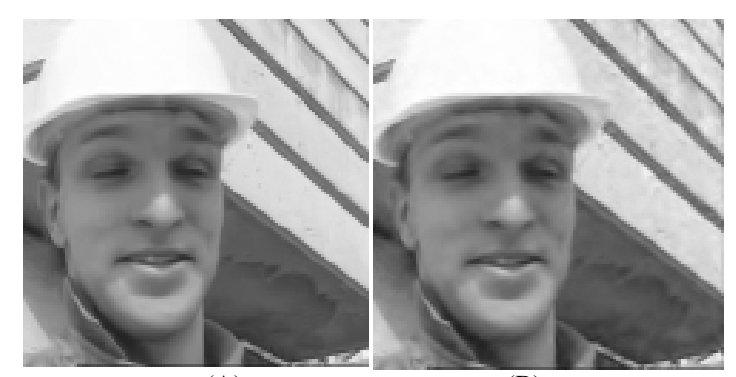

(A)

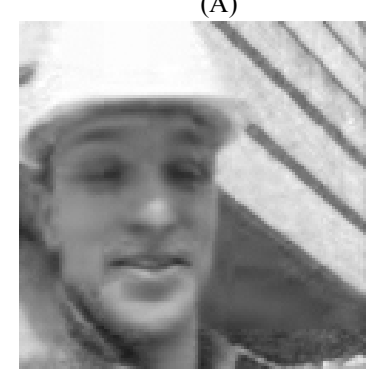

(C)
(B)

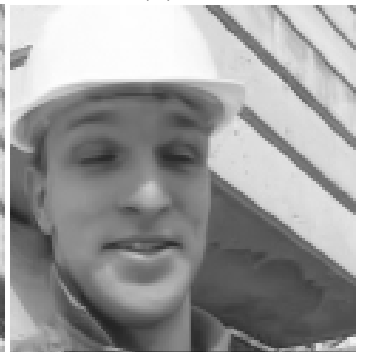

(D)
FIGURE III. THE SUBJECTIVE VISUAL COMPARISON CHART OF FOREMAN'S SECOND FRAME RECONSTRUCTED BY DIFFERENT MATRIX WHEN THE SUBRATE IS 0.4: (A) ORIGINAL FRAME; (B) BERNOULI MATRIX; (C) OSTM; (D) OPTIMIZED OSTM.

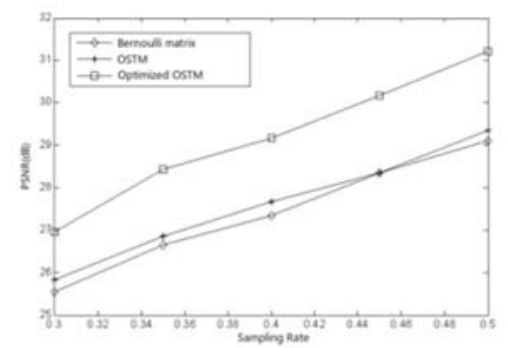

FIGURE IV. PSNR OF RECONSTRUCTED IMAGES FOR DIFFERENT MEASUREMENT MATRICES

In this design of optimization, the Gray complementary sequence is used as the symbol sequence to construct the orthogonal symmetric Toeplitz matrix. Since the Gray complementary sequence has a special autocorrelation property and the pseudo-random cyclic characteristics are added in the process of optimizing the cross-symmetric Toeplitz matrix so that independent elements in the optimized matrix are greatly reduced what has a good effect on reducing the amount of storage and easy implementation of hardware.

\section{CONCLUSION}

Measurement matrix which is the core of compressed sensing, its performance has a direct impact on the complexity of the reconstruction in algorithm and the difficulty of implementation in hardware. In this paper, the optimization method based on orthogonal symmetric Toeplitz measurement matrix by using pseudo-loop structure can be easy to implement in hardware. The optimized matrix is propitious to reduce the independent variables and the storage and computation in distributed compressed video sensing. The simulation results show that the proposed algorithm has low complexity and good image reconstruction quality.

\section{ACKNOWLEDGMENT}

This work is supported by the National Natural Science Foundation of China [61571241], Major projects of Jiangsu Province university natural science research [15KJA510002], and Postgraduate Research \& Practice Innovation Program of Jiangsu Province [SJCX17_0221].

\section{REFERENCES}

[1] R. Calderbank, S. Howard, and S. Jafarpour, "Construction of a large class of deterministic sensing matrices that satisfy a statistical isometry property," Selected Topics in Signal Processing, IEEE Journal of, vol. 4, no. 2, pp. 358-374, April 2010.

[2] Candès E. Compressive sampling[C]// Int Congress of Mathematic. Madrid, Spain, 2006: 1433-1452.

[3] Donoho D, Tsaig Yaakov. Extensions of compressed sensing [J]. Signal Processing, 2006, 86(3): 533-548.

[4] Candès E, Justin R. Practical signal recovery from random projections[C]//IS\&T/SPIE's, 17th Annual Symposium on Electronic Imaging. San Jose, CA, 2005, 54(19): 76-86.

[5] Bottcher A. Orthogonal symmetric Toeplitz matrices [J]. Complex Analysis and Operator Theory, 2008, 2(2): 285-298.

[6] K. Li, C. Ling, and L. Gan, "Statistical restricted isometry property of orthogonalsymmetric toeplitz matrices," in Information Theory Workshop'09. IEEE, Oct. 2009, pp. 183-187.

[7] S. Chatterjee, "Stein's method for concentration inequalities," Probability Theory Related Fields, vol. 138, pp. 305-321, 2007.

[8] Chen Chen, Eric W. Tramel and James E. Flower, Compressed-Sensing Recovery of Images and Video Using Multihypothesis Predictions, 2011 Conference Record of the Forty Fifth Asilomar Conference on Signals, Systems and Computers (ASILOMAR), Pacific Grove, CA, USA, 2011, pp. 1193-1198. 